\title{
RefLEXIONES EN TORNO A LA SITUACIÓN DE LA INVESTIGACIÓN TURÍSTICA
}

\author{
Maribel Osorio García \\ Facultad de Turismo y Gastronomía, \\ Universidad Autónoma del Estado de México. \\ maribelosorio2@gmail.com \\ Maximiliano E. Korstanje \\ Facultad de Ciencias Económicas, \\ Universidad de Palermo. Buenos Aires \\ maxikorstanje@hotmail.com
}

\begin{abstract}
En el artículo que se ofrece, se discute críticamente los desafíos y dificultades que presenta la investigación turística para su madurez científica, señalando sus limitaciones epistemológicas, temáticas y metodológicas, con la intención de instalar una discusión y una plataforma de pensamiento que permita a los epistemólogos latinoamericanos hacer su aporte en la esfera de una epistemología turística que se reconoce así misma en crisis. Para ello, a través de un ejercicio analítico-reflexivo, se cuestiona la división de su campo de conocimiento en turismo como negocio y estudios turísticos, y se enfatiza la necesidad de contrarrestar la fragmentación de su estudio. Finalmente, se abordan las discusiones de décadas pasadas respecto a qué es el turismo, así como también los aspectos que la propia academia no ha podido corregir.
\end{abstract}

Palabras clave: Turismo, epistemología, investigación, campo de conocimiento, Latinoamérica.

\section{REFLECTIONS REGARDING THE STATUS OF TOURISM RESEARCH}

In the article that is offered, it is critically discussed the challenges and difficulties presented by tourism research for its scientific maturity, pointing out its epistemological, thematic and methodological limitations, with the intention of installing a discussion and a platform of thought that allows epistemologists Latin American countries to make their contribution in the sphere of a tourist epistemology that recognizes itself in crisis. For this, through an analytical-reflexive exercise, the division of its field of knowledge in tourism as business and tourist studies is questioned and the need to counteract the fragmentation of its study is emphasized. Finally, we discuss the discussions of the past decades regarding what tourism is, as well as aspects that the academy itself has not been able to correct.

Keywords: Tourism, epistemology, research, field of knowledge, Latin America.

Fecha de recepción: 5 de marzo de 2017. Fecha de aceptación: 15 de abril de 2017.

CÓMO CITAR: Osorio-García, M. y Korstanje, M. E. (2017). Reflexiones en torno a la situación de la investigación turística.

Dimensiones Turísticas, 1(1), 13-30. https://doi.org/10.47557/JJLL9743 


\section{Introducción}

$\mathrm{L}$

a posición del turismo en el contexto mundial es vanagloriada año con año por la Organización Mundial del Turismo al señalar el crecimiento constante de sus indicadores básicos (Ilegadas internacionales, derrama económica, aportación al producto interno bruto y número de empleos, entre los más comunes), reconociéndola como la cuarta actividad de exportación más importante (World Tourism Organization, 2015, p. 14), solo superada por las industrias de los combustibles, la química y la alimentaria. El turismo es materia de alabanza no únicamente para los sectores económicos, sino para los políticos en campaña que lo toman como un aspecto positivo de su gestión (Schlüter, 2008). Por otro lado, no menos cierto es que el turismo en cuanto disciplina académica no solo ha acaparado la atención de muchas universidades, también ha experimentado un incremento sustancial en tesis doctorales, libros, congresos y programas (Brohman, 1996; Xiao y Smith, 2006; Tribe, 2006). Sin lugar a dudas, tal crecimiento va acompañado por un aumento sistemático en la cantidad de visitantes que reciben año con año los principales destinos mundiales (Hall y Lew, 2009). Este rápido y vertiginoso crecimiento de la industria turística ha generado una ruptura entre las visiones de fenómeno social y de negocio de la actividad (Guzman, 1986; Crick, 1989; Getino, 2002; Korstanje, 2008; Monterrubio, 2011; Korstanje, Mustelier y Herrera, 2016).

En los últimos años ha surgido una discusión, por demás interesante, sobre la naturaleza del turismo, y se encuentra lejos de cerrarse (Dann, 2002; Gastal, 2006). Para algunas voces, el turismo es un fenómeno dual, en el sentido de que sus manifestaciones son tanto positivas como negativas, con repercusiones de diversa índole (económicas, sociales, políticas, culturales, etc.) y en distintas dimensiones (temporales, espaciales y sociales), cuya expresión masiva se desvela en el siglo pasado (en la modernidad, según Urry, 2004; Mazón, 2001 y Krippendorf, 1995), pero cuya evolución tiene raíces históricas de mayor alcance (Dumazedier, 1971; Turner y Ash, 1991). Por el contrario, para otros, el turismo se encuentra centrado en una matriz productiva que obedece a un sistema económico que le precede y obviamente le es propio (Ritzer y Liska, 2002). Para estos estudiosos, el turismo no solo debe ser comprendido como una actividad comercial sino además como una pieza de ingeniería orientada a la producción de experiencias y servicios de diversa complejidad (Muñoz de Escalona, 1992, 2000; Vogel, 2001; Eadington y Redman, 1991; Vanhove, 2011; Ascanio, 2014).

No obstante, como fuese denunciado por Tribe (2000) y Thirkettle y Korstanje (2013), la producción y el conocimiento científico del turismo se han fragmentado de una manera en la que aún es imposible una cabal comprensión del fenómeno. Sin duda, los estudios en turismo han crecido en tamaño y volumen. Se puede tomar como referencia la información que proporciona la base de datos Scopus, en la cual, al 2015, el número de textos científicos ascendía a 49 344; de estos, menos de una quinta parte (1.6\%) se publicó antes del año 2000; la tercera parte (34 \%) en la década del 2000, y casi la mitad (50 \%) en lo que va de la presente década, lo que sugiere que las publicaciones científicas en turismo han estado en ascenso alcanzando su zenit en la última década (Scopus, 2015). 
Para la región de Iberoamerica, la base de datos de Scielo reportó un acervo de 948 textos científicos sobre turismo, cuya proporción se conforma de una centésima parte (1\%) publicados antes del año 2000; una tercera parte (35\%) en la década del 2000, y casi dos terceras partes (64\%) en lo que va de la presente década. Es claro que el interés por investigar este fenómeno se ha expandido recientemente, aunque cabe señalar que aún no presenta los alcances de otros campos de conocimiento con igual "antigüedad" (siglo XIX), como es el caso de la comunicación, que registra 1372653 textos científicos para el mismo periodo.

Pese al gran incremento en su producción de conocimiento, ello no se ha traducido en la unificación de un objeto claro, en una epistemología unificada que permita a los turismólogos las bases sólidas para la maduración de su disciplina. En este sentido, los abordajes sistémicos desde la complejidad han dado luz hacia una cabal comprensión del fenómeno (Osorio, 2007).

Así, en la presente pieza de revisión teórica, no solo se discute críticamente los desafíos y las limitaciones de la epistemología del turismo, sino que se argumentan aquellas fallas que han llevado a la no-maduración del turismo como opción seria frente a otras disciplinas. Para ello es necesario abordar las discusiones de décadas pasadas respecto a la naturaleza del turismo, además de comprender los aspectos negativos que la propia academia no ha podido corregir. Sin que ello revista una crítica a algún exponente en particular o corriente teórica, mediante un ejercicio analítico-reflexivo, ${ }^{1}$ el propósito del presente trabajo se orienta a instalar una discusión y una plataforma de pensamiento que permita a los epistemólogos latinoamericanos hacer su aporte en la esfera de una epistemología turística que se reconoce a sí misma en crisis.

\section{Caracterización de la investigación turística}

En principio, se realiza un acercamiento a los aspectos epistemológicos, temáticos y metodológicos de su producción científica, para posteriormente plantear la discusión. Si bien la presente pieza de revisión se trata de un ensayo, el cual muchas veces esquiva a una definición o pronunciación metodológica más precisa, nos hemos centrado en la posición de Serres (1991) y Latour (2007), así como en los aportes de David Harvey (1998), quienes señalan una fragmentación creciente entre las diferentes disciplinas respecto al conocimiento que estas producen. De hecho, advierte Harvey, el conocimiento y la epistemología no pueden ir disociados de las matrices de producción económica.

La dispersión de las epistemologías ha sido un resultado histórico de la introducción de la relatividad como eje discursivo fundante de la ciencia. Si el concepto de realidad, como

\footnotetext{
${ }^{1}$ Como procedimiento metodológico se acudió al método analítico como análisis del discurso, en el sentido que lo refieren Lopera, Ramírez, Zuluaga y Ortiz (2010), considerando tanto una caracterización de los elementos constitutivos de la investigación turística, como la crítica y contrastación de su consistencia y eficacia. Se complementa con una postura crítico-reflexiva, para la valoración de su situación y la necesidad de producir conocimiento sobre nuevos paradigmas epistemológicos.
} 
había sido imaginado por la Escuela de Viena, sostenía una verdad inmutable, duradera y única a la cual cada persona podía acceder con el verdadero proceso (Axtell, 2000; Musgrave, 1993; Paul, 2000), no menos cierto es que la introducción de la relatividad iniciada por el pragmatismo sentó las bases para una ruptura trágica con el concepto de realidad, el cual desembocó en muchas realidades paralelas (Rorty, 1982).

Como bien advierte Harvey, la introducción de la multirrealidad converge con la ruptura de la economía de escala originada luego del embargo petrolero de Medio Oriente durante el conflicto árabe-israelí. En ese contexto, Occidente no solo experimentó un estancamiento económico sin precedentes en sus matrices productivas, sino que comprendió la necesidad de segmentar los productos, abandonando así la producción a escala que había caracterizado la época fordista.

Ese mismo proceso de descentralización económica se ha dado en los estudios epistemológicos considerando el lema "cada persona es un mundo" como parte fundante de la epistemología posmoderna (Harvey, 1998). En tal escenario, resulta importante soslayar que muchas disciplinas nacidas desde 1970 han experimentado una fuerte fragmentación en sus respectivos corpus teóricos así como problemas sustanciales para definir su objeto de estudio. En este entendimiento, el sentido de transdisciplinariedad puede oscurecer más de lo que aclara y, por ende, se hace necesaria una mayor discusión del problema que trascienda las quejas de Tribe (Korstanje, 2013b).

Es relevante, por tal motivo, resaltar las contribuciones de Jost Krippendorf en la construcción de una nueva epistemología del turismo que lo contemple como un ritual de pasaje, un mecanismo de evasión y revitalización onírica que escapa a la modernidad o a una cultura en particular. Como institución milenaria, tal vez con otros nombres, la relación entre el turismo y la fase productiva de cada grupo humano revela similitudes entre la vigilia y el sueño. La realidad que se proyecta en el mundo del turismo es onírica, en el sentido de que es fabricada por un orden precedente. Como el sueño, su función radica en garantizar la funcionabilidad del sistema revitalizando las frustraciones sucedidas durante el trabajo (Korstanje, Bridi y Cappellano dos Santos, 2015).

\subsection{Aspectos epistemológicos}

Las divergencias en el estudio del turismo son comunes. Recuérdese las posturas de Jost Krippendorf y Dean MacCannell sobre el tema. Combinando la teoría de la prensa hidráulica formulada por Sigmund Freud, quien sostenía a la estructura psíquica como una represa que contiene una fuerza que da como resultado diversos procesos, Krippendorf consideraba al turismo como una válvula de escape común a muchas culturas y épocas. Sus avances en estudios psicológicos y culturales lo llevan a confirmar que el turismo revitaliza aquellas frustraciones ocurridas durante la época de trabajo y a tal efecto permite que la sociedad se mantenga unida. En perspectiva, cada sociedad desarrollaría sus propias formas de turismo acorde a una matriz productiva que le antecede. 
Krippendorf no solo esboza una teoría “insolente" sobre los paradigmas económico-céntricos que imperaban por su época, sino que sienta las bases para la construcción de una "conciencia turística" (Krippendorf, 1975, 1982, 1986, 1987a, 1987b, 1989, 1993, 1995). No obstante, otros estudiosos como el antropólogo americano Dean MacCannell han sostenido una tesis totalmente contraria. Reconociendo que el turismo tenga un rol integrador dentro de la cultura, MacCannell sugiere que su nacimiento se encuentra supeditado al avance de la era industrial. Si para las culturas nativas el tótem era el elemento integrador y central de la vida política y social, la misma función la cumple el turismo en las sociedades secularizadas. A razón de ello, huelga decir, el turismo no solo revitaliza las frustraciones individuales sino que pone al sujeto en un paisaje ficticio que sustenta la explotación capitalista. En otras palabras, el turismo sería visto para los ojos de MacCannell como un mecanismo de ideología que legitima culturalmente las bases de la explotación capitalista sobre la fuerza de trabajo (MacCannell, 1973, 1976, 2001).

También es conocida la controversia suscitada entre Leiper (2000) y Tribe (2000) en torno al estatus científico del turismo. Si bien ambos desde diferentes perspectivas han aportado a la epistemología del turismo, sus posiciones pueden estudiarse como divergentes. Mientras Leiper declaraba que el turismo es una disciplina científica emergente debido a la gran cantidad de instituciones, escuelas, tesis, libros y artículos científicos generados sobre él, Tribe debatía que ninguno de los aspectos anteriores le da a este el carácter de ciencia, y que al carecer de objeto de estudio y método propio no alcanza tal condición. Esa discusión no ha sido saldada.

A partir de esta divergencia, varios investigadores se han demarcado por colocar al turismo como un "campo de conocimiento" (Jamal y Robinson, 2009) integrado convencionalmente por dos conjuntos de estudios: Ios administrativos, que abordan al turismo como negocio (tourism managment), y los turísticos, que lo tratan como un fenómeno social (Sharpley, 2011). Al igual que otros investigadores (Ren, Pritchard y Morgan, 2010), esta división se considera una alternativa incompleta y poco fundamentada, entre otras razones porque ignora al conjunto de ciencias naturales y exactas que están estudiando al turismo, pero se reconoce que remarca muy bien el acentuado peso que ha tenido su producción científica bajo la concepción económico-administrativa de la rentabilidad capitalista, que le ha dado su dominante énfasis positivista.

El señalamiento del predominio positivista ha sido hecho por muchos epistemólogos del turismo (Tribe, 2006; Panosso, 2007; Gómez, 2012; Campodónico y Chalar, 2010; Arias, 2012; por mencionar algunos), reconociendo cierto avance en la utilización de los paradigmas pospositivistas (teoría de sistemas complejos), pero enfatizando la necesidad de desarrollar mucho más los enfoques antipositivistas, como las posturas críticas (Gómez, 2012; Castillo, 2011; Panosso y Castillo, 2010) y los estudios fenomenológicos (Panosso, 2007) y hermenéuticos (Conde, 2015). En todo caso, investigar el turismo desde los paradigmas referidos nos revela una realidad distinta de su concepción instrumentalista y hace patentes las contradicciones y los significados que subyacen en su práctica. Si bien tales aportaciones han enriquecido su conocimiento, también han contribuido a su "caos 
epistemológico" (Thirkettle y Korstanje, 2013), al ampliar la fragmentación de sus saberes científicos, volviendo más difícil un encuadre de sus conocimientos (Harrison, 2007). Una evidencia de lo anterior es su amplia diversificación temática.

\subsection{Aspectos temáticos}

En distintos documentos (Osorio y López, 2012; Universidad Nacional Autónoma de México, 2007) se ha expuesto un panorama de las tendencias de la investigación turística en el contexto internacional, recuperando las aportaciones de Jafari $(1994,2005)$, Cohen (cit. en Apostolopoulos, Lerivadi y Yianakis, 2002, pp. 53-64), Xiao y Smith (2006, 2007), Ballantyne, Packer y Axelsen (2009), y Sharpley (2011, p. 55). Con la revisión de diez años de publicación (de 1994 a 2004) de 12 de los más destacados journals especializados en turismo, Ballantyne et al. (2009) elaboraron un listado de 21 temas sobre la producción turística, el cual es actualizado por Sharpley para un periodo de cinco años (de 2005 a 2010) mediante la revisión de cuatro de esos journals (cuadro 1).

Cuadro 1. Tendencias temáticas de la investigación turística (porcentajes)

\begin{tabular}{|lcc}
\hline \multicolumn{1}{|c}{ Temas } & $\begin{array}{c}\text { Ballantyne et al. } \\
(1994-2004)\end{array}$ & $\begin{array}{c}\text { Sharpley } \\
(2005-2010)\end{array}$ \\
\hline 1. Turistas/visitantes & 11 & 16.5 \\
\hline 2. Destinos & 9 & 9.5 \\
\hline 3. Planificación turística & 9 & 3.9 \\
\hline 4. Mercadotecnia & 8 & 10.1 \\
\hline 5. Turismo cultural & 6 & 4.6 \\
\hline 6. Economía & 6 & 8.7 \\
\hline 7. Impactos turísticos & 6 & 2.7 \\
\hline 8. Tendencias turísticas & 6 & 1.9 \\
\hline 9. Investigación y metodología del turismo & 5 & 7.9 \\
\hline 10. Hospitalidad & 4 & 3.2 \\
\hline 11. Ecoturismo & 4 & 1.5 \\
\hline 12. Desarrollo sustentable & 4 & 2.5 \\
\hline 13. Eventos especiales & 3 & 1.1 \\
\hline 14. Transportación & 3 & 1.1 \\
\hline 15. Administración & 2 & 8.7 \\
\hline 16. Recursos humanos & 2 & 2.9 \\
\hline 17. Interpretación ambiental & 2 & 1.4 \\
\hline 18. Política turística & 2 & 3.7 \\
\hline 19. Educación y capacitación turísticas & 2 & 2.6 \\
\hline 20. Turismo de negocios & 1 & 0.6 \\
\hline 21. Deportes y ocio & 1 & 0.9 \\
\hline 22. Comunidad, anfitriónes y huéspedes & & 3.7 \\
\hline
\end{tabular}

Fuente: Elaboración propia a partir de la información de Ballantyne et al. (2009) y Sharpley (2011). 
El listado, aunque varía en su criterio de clasificación (lo mismo hay disciplinas científicas, que modalidades de turismo), refiere que el foco central de atención de la investigación turística ha sido el turista, como actor clave del desplazamiento y “detonador" de los distintos procesos generados por la movilidad. Así también denota que los temas de destinos y mercadotecnia se mantienen como relevantes y con tendencia al alza, en tanto que aquellos ligados a planificación, desarrollo turístico sustentable y tendencias están a la baja. No hay que perder de vista que los journals incluidos en los listados solo están revelando la información de las revistas especializadas en turismo, por lo que en realidad muestran que la investigación turística “de mayor impacto" está acentuando su concepción del turismo como negocio, lo que es consistente con la hiperacumulación capitalista que vive el mundo contemporáneo (Harvey, 1998).

No obstante, se debe ubicar esta apreciación en su justa dimensión, ya que el abordaje del estudio social del turismo, como lo revela la clasificación temática de Scopus (gráfica 1), es un tema de mayor profusión que el solo visualizado en los mencionados journals de turismo.

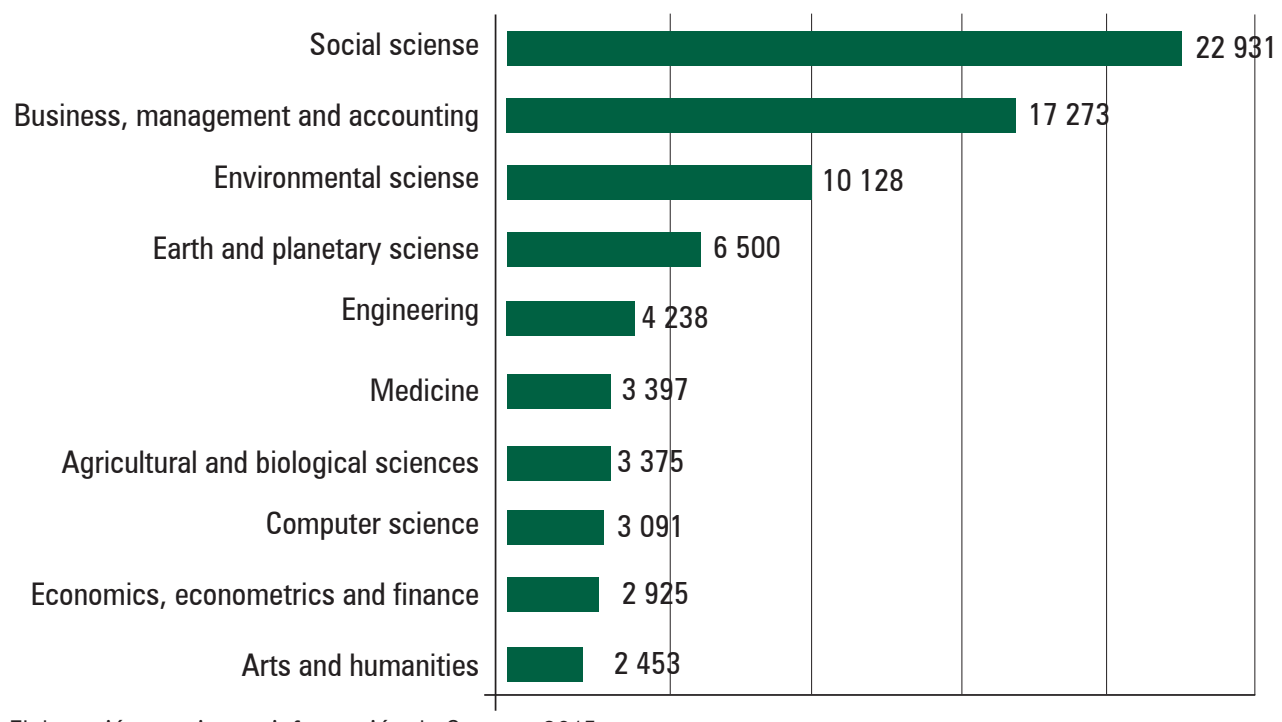

Fuente: Elaboración propia con información de Scopus, 2015.

GráfICA 1. Textos científicos de turismo por área de conocimiento.

No obstante que la producción de la investigación turística latinoamericana se muestra en una cantidad limitada en el contexto internacional (Peral y Gil, 2013), cuenta ya con una trayectoria que revela su propia tendencia temática. Según las aproximaciones realizadas por Osorio (2016), exhibe un especial énfasis por los temas territoriales vinculados con la sustentabilidad y el desarrollo local, como un eje temático dominante que expresa los focos de atención de su contexto. Asimismo, González y Palafox (2014) han mostrado que los temas sociales tienen una presencia mayor en la investigación turística iberoamericana que la esperada, configurando una interesante línea de investigación sociológica en 
el turismo. Entonces, varios indicios permiten inferir que la investigación turística latinoamericana ha trazado sus preferencias temáticas de manera diferenciada y con un fuerte énfasis social.

\subsection{Aspectos metodológicos}

Las revisiones efectuadas por distintos estudiosos del turismo (Koc y Boz, 2014; Ballantyne, Packer y Axelsen, 2009; Jennings, 2009) coinciden en señalar el dominante peso del uso de metodologías cuantitativas para la construcción de su conocimiento. Según Koc y Boz (2014, pp. 10-11), la encuesta por cuestionario es la técnica más recurrente, complementada con relativa frecuencia por la entrevista. El empleo de las metodologías cualitativas aún tiene un peso poco significativo en la investigación turística, en donde las entrevistas y el análisis de contenido son las más usuales. Lo anterior es consistente con el dominio del paradigma positivista y el enfoque instrumentalista que ha tenido la investigación turística en el nivel internacional.

Se enfatizan tres debilidades metodológicas que los diversos autores han indicado: la triangulación metodológica, entendida como una estrategia de solidez para la generación de hallazgos y resultados, es poco trabajada en la investigación turística -prácticamente en solo tres de cada diez investigaciones-. Un segundo elemento estriba en que la gran mayoría de las investigaciones son estudios de caso, realizados en un solo organismo o localidad, contando con pocos estudios replicados que permitan comparaciones sobre sus resultados. El tercer punto radica en que la mayoría de las investigaciones son estudios sincrónicos, por lo que se desconoce la evolución y cambios de comportamiento del objeto de estudio a lo largo del tiempo. Estas características han limitado la calidad de la investigación turística, dando pie a señalamientos frecuentes en torno a la necesidad de fortalecer su rigor metodológico.

No se sabe de algún estudio que otorgue información sobre los aspectos metodológicos en Latinoamérica, pero se infiere que la situación es muy similar a lo expuesto, e incluso más acentuada respecto al nivel descriptivo de los resultados de investigación.

\section{Desafíos de la investigación turística}

Desde la década de los setenta, se ha venido señalando que la investigación turística debe transitar hacia un ejercicio interdisciplinario como la mejor opción para superar la fragmentación disciplinaria, ya que ofrece la posibilidad de mediar distintos cuerpos de conocimiento y alcanzar una hibridación de conceptos y prácticas para una coherente, consistente y adecuada explicación del fenómeno turístico. Esta es la base de la argumentación de Darbellay y Stock (2012, p. 454) para recalcar que el camino pertinente a seguir para su consolidación científica no estriba en una organización paradigmática de su conocimiento, 
ni tampoco en su conformación como un sistema autónomo y unificado de conocimientos, sino en un proyecto cognitivo en el que las dimensiones turísticas de la sociedad sean el foco de atención de las aproximaciones interdisciplinarias. Aunque por supuesto se está de acuerdo con la propuesta interdisciplinaria, a más de 30 años de su "puesta en uso", se aprecia que este planteamiento ha tenido más resonancia que implementación, no solamente por el grado de dificultad que implica, sino porque se continúa trabajando sobre plataformas de limitado alcance.

Frente a fenómenos de una realidad cambiante, incierta e inestable, la pérdida de capacidad explicativa y comprensiva de los planteamientos teórico-convencionales de las ciencias sociales, conformados a partir de una epistemología modernista, ha generado la búsqueda de nuevos paradigmas para comprender y acceder a lo social, siendo las corrientes de pensamiento como la fenomenología, la hermenéutica, el marxismo contemporáneo, el constructivismo y los sistemas complejos las nuevas formulaciones de la llamada epistemología posmoderna para llevar a cabo tal tarea (Arellano, 2015; Aguilar, 2010; González y Arzate, 2015; Massé, 2013).

Para el caso del turismo, se reconoce que la hermenéutica y los sistemas complejos han enriquecido particularmente las explicaciones interdisciplinarias sobre él. El pensamiento hermenéutico ha aportado un amplio estudio cualitativo acerca del sentido, las motivaciones y los imaginarios inmersos en la movilidad turística; las teorías de sistemas complejos han incorporado el entendimiento de las escalas, los sistemas societales y las relaciones sociedad-naturaleza que influyen su dinámica. No obstante, se señala aquí que ambas propuestas tienen sus respectivas dificultades: la comprensión hermenéutica del turismo ha extremado la polisemia de los análisis subjetivos y relativizado la condición de los elementos materiales; y las teorías de sistemas complejos carecen de suficientes herramientas conceptual-metodológicas para investigar la causalidad heterogénea (social y natural) del fenómeno turístico.

Si las teorías mencionadas están entre las propuestas de vanguardia más sobresalientes en el estudio del turismo, siguiendo la ironía de Sharpley (2011, p. 49) cuando pregunta en dónde nos encontramos ahora en tal estudio, se tiene que admitir su respuesta: "estamos en donde siempre hemos estado". Así entonces, desde el punto de vista de quienes escriben, el desafío del estudio científico del turismo no solo estriba en crear nuevos conceptos que resuelvan la hibridación interdisciplinaria, o en aplicar metodologías mixtas (cuanticualitativas) en estudios diacrónicos, sino en superar la herencia de las explicaciones fragmentadas derivadas de la epistemología modernista y explicar integralmente su heterogénea causalidad. Esto implica reconocer la compleja empiricidad del turismo e integrarla en la explicación de su objeto de estudio, y es en este punto, en el reconocimiento de la empiricidad de los fenómenos, donde falla la propia epistemología posmoderna en los distintos campos del conocimiento científico (Arellano, 2015). La alternativa para afrontar ese reto consiste en explorar otras epistemologías que permitan incorporar y traducir los integrados elementos de la realidad turística, hasta ahora representados de modo aislado (Korstanje, 2013b). 
Una alternativa es la propuesta que Korstanje, Bridi y Cappellano dos Santos elaboraron recientemente como una teoría por demás particular que define al turismo como un ritual de pasaje el cual se encuentra orientado - de la misma forma que el sueño- a una dimensión lúdica y onírica, en donde el turista juega a ser quien en realidad no es. Esta realidad, lejos de ser ficcional y alienatoria, permite la verdadera revitalización del lazo social, del mismo modo que el sueño permite el correcto descanso del cuerpo preparándolo para la vigilia (Korstanje et al., 2015). El turismo, según esta concepción, rompe con la realidad creando un mundo de ensueño donde la regla cede frente al juego. El viaje opera como forma de ruptura entre lo conocido de la vigilia y lo desconocido que ofrece el mundo de los sueños. El sujeto es sometido a un paisaje que toma partes de la realidad pero las ordena

acorde a una matriz onírica. Dentro del sistema social se producen clivajes no solo entre los diferentes actores entre los subsistemas pertinentes. El turismo opera dentro del ocio, el cual es parte del subsistema onírico cuya función central es evitar la disgregación social. Por ese motivo, y lejos de ser una actividad baladí, el turismo debe ser comprendido como un factor clave en la configuración del ethos social.

\section{Dificultades en el camino}

Recientemente, varios estudios críticos han apuntado a la crisis sustancial que experimenta la epistemología del turismo en habla inglesa. Entre los diversos factores se encuentra una academia que no dialoga con las bases o los investigadores, creando de esa manera pequeñas islas de conocimiento dispersas e imposibilitadas para integrarse como un corpus teórico completo. Como bien ha expuesto John Tribe (2010), la International Academy for the Study of Tourism (AIST) ha promovido incansablemente la investigación aplicada aun cuando no ha aportado bases epistemológicas firmes respecto de cómo se debe investigar. Ello no solo ha generado una fragmentación innecesaria en la producción académica, sino también que los investigadores se cerrasen en islas, redes totalmente desconectadas unas de otras. En este sentido, Graham Dann ha realizado una crítica exhaustiva que amerita ser discutida en la presente sección. Dann entiende que la AIST se encuentra formada en su mayoría por angloparlantes, y que este hecho no permite incorporar otras voces, culturas o idiomas con el fin de expandir el curso de acción. Como resultado, la investigación aplicada se acomoda para validar los prejuicios preexistentes en aquellas naciones de habla inglesa sin lograr un entendimiento respecto a otras formas de comprender el mundo (Dann, 1996, 2009, 2011).

A estas limitaciones, y en segundo lugar, se debe agregar que los estudios epistemológicos vigentes han adquirido gran parte de su contenido del positivismo y, en razón de tal, creen que preguntar es el único modo de estudiar un fenómeno. En este tenor, Korstanje ha desarrollado una posición respecto a los problemas metodológicos reales de las investigaciones en turismo. Por un lado, se considera al turista como el eje sobre el cual debe ponerse la atención. La mayoría de estos estudios enfatizan en la posición del turista, o su manera de ver tal o cual fenómeno, olvidando que muchas veces hay otras formas de comprensión. 
Por otro lado, se introducen metodologías intrusivas basadas en cuestionarios, entrevistas formales o informales que llegan a oscurecer más que aclarar. En parte, todas estas metodologías, así como el positivismo y la fenomenología, olvidan que en muchas ocasiones las personas mienten para proteger sus intereses, o inventan historias para agradarle a los investigadores, mientras que en otros casos sencillamente desconocen su vida emocional interna. Como bien se ha observado, si un turista consultado en un museo pondera como positivo el consumo de cultura, eso simplemente es un constructo colectivo que el turista ha internalizado, no debe ser considerado como una verdad ontológica (Korstanje y George, 2015; Korstanje et al., 2016; Korstanje, 2013a; 2013b).

En tercer y último lugar, la presión por publicar en revistas de gran impacto ha mellado hondo en muchos círculos de investigadores creando una carrera cuasi-darwinística por la exclusividad, pero alejando a las publicaciones no solo de la calidad académica sino además de las bases epistemológicas necesarias para la consolidación de la disciplina. En este aspecto, se genera una guerra entre las mismas revistas en donde las de acceso gratuito quedan relegadas en los peores rankings, mientras que aquellas donde el contenido es de pago (pay-for journals) son situadas en la cumbre de la pirámide, precisamente por las compañías editoriales que formulan tales índices. Como resultado, investigadores provenientes de naciones periféricas desactualizan sus citas bibliográficas ya que por desigualdades económicas preexistentes no tienen el capital para pagar por los artículos, o simplemente se ven retraídos a citar a aquellos autores de habla inglesa que se consideran de culto. Esta verdadera barrera económica produce una distancia mayor que no permite el diálogo multidisciplinar entre los diferentes continentes, llevando a lo que proclamaba Douglas Pearce en su célebre “internacionalización” del conocimiento turístico.

En un trabajo publicado en una de las mejores revistas de habla hispana, Estudios y Perspectivas en Turismo, Pearce enfatiza que América Latina debe copiar el ejemplo de Asia, continente que ha desarrollado un gran lazo de intercambio con Estados Unidos e Inglaterra. Los investigadores chinos han podido insertarse en el mercado editorial anglosajón debido a que han adoptado al inglés como lengua franca. Pero no únicamente eso, Pearce redobla la apuesta y explica que los autores latinoamericanos mantienen un impacto bajo respecto a las citas en comparación con sus colegas de habla inglesa por varios motivos, aunque subraya dos en particular. El primero se corresponde con la falta de interés de los anglosajones por los estudios en español, y el segundo se refiere a que las revistas de turismo no publican muchos estudios tomando a América Latina como estudio de caso. ¿Cómo resuelve Pearce esta dicotomía?

El proceso de internacionalización del turismo implica no solo elevar los actuales estándares de calidad, sino además fijar políticas claras y conjuntas en materia de investigación. A partir de que la conectividad y el mejoramiento en las condiciones competitivas de los destinos exigen proporciones o umbrales de gobernanza mayores, los investigadores deben proveer el conocimiento necesario a quienes toman a diario las decisiones. Desde el momento en que el inglés no es la lengua materna de los investigadores hispanohablantes, deben mantener un lazo estrecho con lo producido en Estados Unidos y las naciones de habla inglesa. Pearce (2013, p. 916) dice abiertamente: "grandes países como los EEUU 
van a tener una gama más amplia de posibilidades de publicación, intercambiar ideas y un más amplio grupo de evaluadores a los cuales recurrir. En contraste puede ser que no existe aún una masa crítica de investigadores en turismo en países más pequeños donde la investigación está en una etapa más temprana de desarrollo".

Por su parte, Korstanje (2014) reacciona a estos comentarios con una pieza publicada en la revista brasileña Rosa Dos Ventos donde queda en evidencia no solo el etnocentrismo anglosajón, sino las deficiencias de una epistemología que, lejos de repreguntarse, ya tiene respuestas cerradas para cuestiones que no se han formulado. Korstanje adhiere a la tesis que la investigación en habla inglesa tiene un gran tinte economicista, y considera al turismo como un objeto productor de valor. El turismo es concebido únicamente como objeto, debido al valor que puede producir en el territorio. Esta mirada sesgada ha generado que los investigadores estén más interesados en aquellos factores que atentan contra la industria, en lugar de focalizar sus esfuerzos en comprender el origen de tales amenazas.

En segundo lugar, los colegas de habla inglesa ven en la experiencia la unidad de negocio que puede ser segmentada con el fin de optimizar los negocios o la performance del destino. De este modo, los intentos de planificación dan como resultado la proliferación de nuevos destinos, los cuales organizados bajo nuevos horizontes temáticos convocan a una red mayor de investigadores. En otras palabras, si los padres fundadores de la disciplina se encontraban abocados a responder qué es el turismo, esta pregunta ha sido remplazada por la de cuántas formas nuevas de turismo podemos descubrir. Al turismo clásico se le suman el rural, cultural, negro, blanco, etc. Esta diversificación de productos, los cuales son conducentes a la lógica del mercado, por cuanto mayores productos implica mayores ganancias, ha generado una fragmentación sin precedentes que hoy pone a la investigación en turismo en crisis (Korstanje, 2014), como lo muestra la tendencia expuesta sobre las temáticas turísticas en los últimos años y los peligros que ello conlleva.

\section{Reflexiones finales}

Para que la maduración de la ciencia turística sea una realidad, la pregunta para qué sirve el turismo debe ser remplazada por la de qué es el turismo. Como se mencionó en este texto, dos posturas antagónicas se han debatido históricamente para imponer su propia visión del turismo. La posición sociocultural hace foco en el turismo como una institución social, el cual no solo cumple un rol protagónico en la sociedad, sino en la confianza del ciudadano hacia sus instituciones, que es la base angular de la sociedad.

Por otro lado, la tradición económico-céntrica, la cual se enfoca particularmente en el marketing y el management, define al turismo como un productor de valor, una pieza productiva ejemplar que permite mejorar la vida de las poblaciones locales. Visto el problema desde este ángulo, si para la primera el turismo debe ser un objeto de estudio mutuamente excluyente por sus propias definiciones, para la segunda, las definiciones sobre qué es y cómo opera el turismo se tornan secundarias acorde a lo que instrumentalmente puede hacerse con este. En otras palabras, a la pregunta qué es el turismo, la cual ha iluminado 
la posición de sociólogos, antropólogos y científicos sociales de diversos países y culturas, se le suma una en el polo opuesto: para qué sirve el turismo.

En un libro de publicación reciente, titulado Tourism Research Frontiers, Chambers y Rakic (2015) Ilaman la atención sobre la crisis de sentido que atraviesa actualmente la investigación aplicada en turismo y hospitalidad. Pero lejos de acoplarse a lo que sugiere la plataforma crítica, lo cual implica una total destrucción de toda la teoría turística vigente, las investigadoras británicas buscan opciones alternativas. Muy al contrario de ser este mundo altamente globalizado uno comparable a aquel desde donde los padres fundadores de la disciplina producían teoría, es necesario comprender que los hábitos son construcciones en constante transformación. Se vive en un mundo de compleja y permanente incertidumbre que pone al turista frente a un cambio de cosmología. En este sentido, resulta inevitable modificar los paradigmas básicos sobre los cuales debe apoyarse la investigación.

Con el fin de buscar nuevos objetos de estudio, es importante que los epistemólogos expandan los bordes de la disciplina. Estos cambios deben ser puentes simbólicos -entre lo conocido y lo desconocido- que dialoguen con otras disciplinas. Chambers y Rakic observan que no es la producción o el tamaño de esta lo que le da credibilidad a una disciplina, sino su capacidad discursiva para explicar los fenómenos que intenta estudiar. El choque de paradigmas produce contornos por medio de los cuales las disciplinas convergen, pero este juego posiciona ciertos saberes como hegemónicos mientras que otros son relegados hacia la periferia. Los turistas modernos buscan no solo comoditizar la cultura, sino también objetivar a los nativos acorde a sus propias experiencias. Ya no se puede comprender la sustentabilidad del turismo sin comprender tal lógica, la cual se aplica a la hipermovilidad de las naciones globalizadas. Empero, en lugar de desmantelar todo lo creado, es importante buscar nuevas alternativas teóricas que focalicen en la política del turismo. El poder político extorsiona a la opinión pública generando una atmósfera de desconfianza y miedo sobre el extranjero; una suerte de declive de la hospitalidad promovida por ciertos sectores orientados a fagocitar el nacionalismo.

¿Cuál es el rol del turismo en estos procesos de cambio? En algún sentido, Chambers y Rakic, continuando los objetivos de Jafari (1994), intentan sentar las bases para una epistemología consolidada pero sin destruir lo que ha sido mal apuntalado. En este sentido, el libro no solo no responde a los objetivos que las editoras se plantean, sino que se asienta en toda una teoría que descansa sobre cimientos endebles; un corpus teórico altamente fragmentado que no ha obtenido con claridad su objeto de estudio. Los defensores de la epistemología actual proclaman que el turismo no se ha transformado en disciplina académica consolidada por una cuestión de tiempo. En forma de anécdota vale mencionar que el psicoanálisis se ha situado como una ciencia en apenas 15 años. Sus padres fundadores aportaron una episteme, un método y un objeto definido de estudio. Cada una de esas disciplinas, como la sociología -la cual se ocupa de las normas-, la antropología -que hace lo propio con la cultura- o la geografía -y su estudio del territorio-, ha desarrollado un objeto de estudio. Aquí cabe preguntarse qué estudia el turismo. 
Si bien Chambers y Rakic intentan discutir el problema de la epistemología del turismo desde un nuevo lenguaje, sus intentos no pueden resolverlo. Ellas no parecen interesadas en explicar la fragmentación del conocimiento, ni tampoco en destruir la divergencia cada vez mayor- entre el turismo como producto económico y el turismo como institución social. De ello se deriva que en los años venideros los investigadores y epistemólogos enfrenten verdaderos desafíos que modificarán no únicamente los contornos disciplinarios del turismo sino su naturaleza misma.

Por lo anterior, de nuestra parte se considera necesario insistir en el requerimiento de investigar sobre nuevos paradigmas epistemológicos que superen la fragmentación disciplinaria para el abordaje de su estudio, que ayuden a entender su heterogeneidad y multicausalidad y aprehender su empiricidad desde una perspectiva integral. Como se apuntó con antelación, una alternativa puede ser la plataforma proporcionada por Serres (1991) y Latour (2007) para acercarnos a explicar, nuevamente, la naturaleza y complejidad del turismo, como se ha hecho para otros objetos de estudio (Arellano, 2015).

\section{Referencias}

Aguilar G., M. (2010). La sociología: construcción categorial, objeto y método. Madrid, España: Tecnos.

Apostolopoulos, Y., Lerivadi, S. y Yianakis, A. (2002). The Sociology of Tourism. Londres, Gran Bretaña: Routledge.

Arellano H., A. (2015). Cambio climático y sociedad. Ciudad de México, México: Porrúa.

Arias C., A. E. (2012). Análisis de las visiones del turismo en México. Turismo em Anlisé, 23(2), 286-307.

Ascanio, A. (2014). Turismo como sistema de servicios: Soportes físicos y estrategias. Papers de Turisme, 6(1), 35-50.

Axtell, G. (2000) Introduction. En G. Axtell (ed.), Knowledge, Belief and Character: Reading in Virtue Epistemology (pp. xi-xxix). Boston, Estados Unidos: Rowman \& Littlefield Publishers.

Ballantyne, R., Packer, J. y Axelsen, M. (2009). Trends in tourism research. Annals of Tourism Research, 36(1), 149-152.

Brohman, J. (1996) New directions in tourism for third world development. Annals of Tourism Research, 23(1), 48-70.

Campodónico, R. y Chalar, L. (julio, 2010). Turismo: una ciencia en construcción. En Anais do VI Seminario de Pesquisa em turismo do MERCOSUL-Saberes no turismo: Interfaces. Universidade de Caxias do Sul, Caxias do Sul, Brasil.

Castillo N., M. (2011). Epistemología crítica del turismo ¿qué es eso? Turismo em Anlisé, 22(3), 516- 538.

Chambers, D. y Rakic, T. (eds.). (2015). Tourism Research Frontiers: Beyond the Boundaries of Knowledge. Wagon Lane, Reino Unido: Emerald Group Publishing.

Conde G., N. (2015). Notas sobre el sistema turístico y la hermenéutica del viaje y la hospitalidad. En Daniel Hiernaux N. (coord.), Turismo, sociedad y territorio. Una lectura 
crítica (pp. 11-42). Querétaro, México: Universidad Autónoma de Querétaro.

Crick, M. (1989). Representations of international tourism in the social sciences: Sun, sex, sights, savings, and servility. Annual Review of Anthropology, 18(1), 307-344.

Dann, G. M. (1996). The Language of Tourism: A Sociolinguistic Perspective. Wallingford, Estados Unidos: Cab International.

Dann, G. M. (2009). How international is the International Academy for the Study of Tourism? Tourism Analysis, 14(1), 3-13.

Dann, G. M. (2011). Anglophone hegemony in tourism studies today. Enlightening Tourism. A Pathmaking Journal, 1(1), 1-30.

Dann, G. M. (ed.). (2002). The Tourist as a Metaphor of the Social World. Wallingford, Estados Unidos: Cabi.

Darbellay, F. y Stock, M. (2012). Tourism as complex interdisciplinary research object. Annals of Tourism Research, 39(1), 441-458.

Dumazedier, J. (1971). Realidades del ocio e ideologías. En J. Dumazedier y R. Kaes, Ocio y sociedad de clases (pp. 9-46). Barcelona, España: Fontanella.

Eadington, W. R. y Redman, M. (1991). Economics and tourism. Annals of Tourism Research, 18(1), 41-56.

Gastal, S. (2006). Tiempos postmodernos: Posibilidades para el turismo. Estudios y Perspectivas en Turismo, 15(3), 270-282.

Getino, O. (2002). Turismo: entre el ocio y el neg-ocio. Buenos Aires, Argentina: La Crujía.

Gómez N., S. (2012). Educación superior e investigación turística. Retos, problemas y desilusiones. Guadalajara, México: Universidad de Guadalajara.

González D., A. y Palafox M., A. (2014). Sociología del turismo en español. Revisión exploratoria de artículos publicados en revistas iberoamericanas 2003-2013. Estudios y Perspectivas en Turismo, 23(4), 805-819.

González G., N. y Arzate S., J. G. (2015). Retos de las ciencias sociales y su vínculo con los estudios avanzados. Programas investigativos y problemáticas emblemáticas. En R. Medrano G., A. Santos L. y J. J. Gutiérrez Ch. (coords.), Las ciencias sociales y sus abordajes en los estudios avanzados (pp. 33-50). Ciudad de México, México: Ediciones Eon.

Guzman, L. F. J. (1986). Teoría turística: un enfoque integral del hecho social. Bogotá, Colombia: Universidad Externado de Colombia.

Hall, C. M. y Lew, A. (2009). Understanding and Managing Tourism Impacts: An Integrated Approach. Abingdon, Reino Unido: Routledge.

Harrison, D. (2007). Towards developing a framework for analysing tourism phenomena: A discussion. Current Issues in Tourism, 10(1), 61-86.

Harvey, D. (1998). La condición de la posmodernidad. Buenos Aires, Argentina: Amorrortu.

Jafari, J. (1994). La cientifización del turismo. Estudios y Perspectivas del Turismo, 3(1), 7-36.

Jafari, J. (octubre, 2005). Revapyng old challengers for integrative paradigms. En VII Congreso Nacional y I Internacional de Investigación Turística. Universidad de Guadalajara y Secretaría de Turismo, Guadalajara, Jalisco, México.

Jamal, T. y Robinson, M. (2009). Introduction: The evolution contemporary positioning of tourism as a focus of study. En T. Jamal y M. Robinson (eds.), Tourism Studies (pp. 1-16). Londres, Gran Bretaña: Sage. 
Jennings, G. R. (2009). Methodologies and methods. En T. Jamal y M. Robinson (eds.), Tourism Studies (pp. 672-692). Londres, Gran Bretaña: Sage.

Koc, E. y Boz, H. (2014). Triangulation in tourism research: A bibliometric study of top three tourism journals. Tourism Management Perspectives, 12, 9-14.

Korstanje, M. (2008). Epistemología de la palabra para las ciencias del turismo. Nómadas. Revista Crítica de Ciencias Sociales y Jurídicas, 19(3), 93-111.

Korstanje, M. (2013a). Ciencia de la hospitalidad: un nuevo aporte epistémico para comprender el objeto del turismo. Recuperado de http://www.eumed.net/libros-gratis/2013a/1331/1331.pdf.

Korstanje, M. E. (2013b). Turismus Sistemae: epistemología del viaje onírico. IJSSTH, 1(4), 24-35.

Korstanje, M. (2014). Crítica al concepto de internacionalización de Douglas Pearce. Rosa Dos Ventos, 6(1), 117-120.

Korstanje, M. E. y George, B. P. (2015). The media or the message? An examination of myths as resources to understand the tourism phenomenon. International Journal of Tourism Anthropology, 4(2), 122-141.

Korstanje, M. E., Bridi, G. y Cappellano dos Santos, M. M. (2015). Turismo y ensueño: el intercambio de roles. Papers de Turisme, 58, 80-92.

Korstanje, M. E., Mustelier, L. C. y Herrera, S. (2016). Understanding the indiscipline of tourism: A radical critique to the current. En I. Bregoli y N. Pappas (eds.), Global Dynamics in Travel, Tourism, and Hospitality (pp. 208-222). Hershey, Estados Unidos: IGI Global.

Krippendorf, J. (1975). Die Landschaftsfresser: Tourismus u. Erholungslandschaft [The Landscape Eaters]. Berna, Suiza: Hallwag.

Krippendorf, J. (1982). Towards new tourism policies: The importance of environmental and sociocultural factors. Tourism Management, 3(1), 135-148.

Krippendorf, J. (1986). The new tourist - Turning point for leisure and travel. Tourism Management, $7,131-135$.

Krippendorf, J. (1987a). Ecological approach to tourism marketing. Tourism Management, 8, 174-176.

Krippendorf, J. (1987b). The Holiday-makers: Understanding the Impact of Travel and Tourism. Oxford, Reino Unido: Butterworth-Heinemann.

Krippendorf, J. (1989). Fur einen Anderen Tourimus: Probleme-Perspektiven [For Another Tourism]. Fráncfort del Meno, Fischer: Taschenbuch Verl.

Krippendorf, J. (1993). Auf dem Weg nach Okotopia? [One Way Towards an Eco-activism]. Wien: Picus.

Krippendorf, J. (1995). Freizeit \& Tourismus: Eine Einfuhrung in Theorie und Politiks [Freedom and Tourism]. Berna, Suiza: FIF.

Latour, B. (2007). Nunca fuimos modernos. Ensayo de antropología simétrica. Buenos Aires, Argentina: Siglo XXI Editores.

Leiper, N. (2000). An emerging discipline. Annals of Tourism Research, 27(3), 805-809.

Lopera E., J. D., Ramírez G., C. A., Zuluaga A., M. U. y Ortiz V., J. (2010). El método analítico como método natural. Nómadas. Revista Crítica de Ciencias Sociales y Jurídicas, 25. Recuperado de http://www.redalyc.org/articulo.oa?id=18112179017 
MacCannell, D. (1973). Staged authenticity: Arrangements of social space in tourist settings. American Journal of Sociology, 79, 589-603.

MacCannell, D. (1976). The Tourist: A New Theory of the Leisure Class. Los Ángeles, Estados Unidos: University of California Press.

MacCannell, D. (2001). Tourist agency. Tourist Studies, 1, 23-37.

Massé N., C. E. (coord.). (2013). La complejidad autorreflexiva epistemológica de las ciencias sociales y su diversidad campotemática. Ciudad de México, México: Porrúa.

Mazón, T. (2001). Sociología del turismo. Madrid, España: Centro de Estudios Ramón Areces, S. A.

Monterrubio, J. C. (2011). Turismo y cambio sociocultural. Una perspectiva conceptual. Ciudad de México, México: Plaza y Valdés.

Muñoz de Escalona, F. (1992). Turismo y desarrollo. Estudios Turísticos, 115(1), 23-44.

Muñoz de Escalona, F. (2000). Autopsia del turismo. Málaga, España: Grupo Eumed.net. Recuperado de https://books.google.com.ar/books?hl=es\&lr=\&id=jXBBNK14Jlk$\mathrm{C} \& \mathrm{oi}=\mathrm{fnd} \& \mathrm{pg}=\mathrm{PA} 2 \& \mathrm{dq}=\mathrm{Munoz}+\mathrm{de}+$ escalona\&ots=PausvRyEtv\&sig=bp2I0He0_ WEOF5aVi0oi9UmrR5o\#v=onepage \&q=Munoz\%20de\%20escalona\&f=false.

Musgrave, A. (1993). Common Sense, Science and Scepticism: A Historical Introduction to the Theory of Knowledge. Cambridge, Reino Unido: Cambridge University Press.

Osorio G., M. (2007). El carácter social del turismo: Un análisis sistémico sobre su complejidad. Estudios y Perspectivas en Turismo, 16(4), 464-492.

Osorio G., M. (2016). Revista Estudios y Perspectivas en Turismo: Calidad científica y editorial, temáticas e indicadores bibliométricos. Estudios y Perspectivas en Turismo, 25(4), 539-557.

Osorio G., M. y López L., Á. (2012). Introducción: producción académica reciente en la investigación turística. En M. Osorio y Á. López (coords.), Investigación turística. Hallazgos y aportaciones (pp. 15-22). Toluca, México: Universidad Autónoma del Estado de México/Universidad Nacional Autónoma de México/Academia Mexicana de Investigación Turística/Secretaría de Turismo.

Panosso N., A. (2007). Filosofía del turismo. Teoría y epistemología. Ciudad de México, México: Trillas.

Panosso N., A. y Castillo N., M. (2010). Epistemología del turismo. Estudios críticos. Ciudad de México, México: Trillas.

Paul, R. (2000). Critical thinking, moral integrity and citizenship. En G. Axtell (ed.), Knowledge, Belief and Character: Reading in Virtue Epistemology (pp. 163-176). Boston, Estados Unidos: Rowman \& Littlefield Publishers.

Pearce, D. (2013). Comprometiéndose con el mundo: América Latina y la internacionalización de la investigación en turismo. Estudios y Perspectivas en Turismo, 22(5), 908-925.

Peral, P. P. y Gil, S. M. (2013). Difusión de la investigación científica en turismo. El caso de México. El Periplo Sustentable, 24, 7-40.

Ren, C., Pritchard, A. y Morgan, N. (2010). Constructing tourism research. A critical inquiry. Annals of Tourism Research, 37(4), 885-904.

Ritzer, G. y Liska, A. (2002). McDisneyization and post-tourism. Complementary perspectives on contemporary tourism. En Ch. Rojek y J. Urry (eds.), Touring Cultures. 
Transformations of Travel and Theory (pp. 96-109). Nueva York, Estados Unidos: Routledge.

Rorty, R. (1982). Consequences of Pragmatism: Essays, 1972-1980. Mineápolis, Estados Unidos: University of Minnesota Press.

Schlüter, R. G. (2008). Turismo: una visión integradora. Buenos Aires, Argentina: Centro de Investigaciones y Estudios Turísticos.

Scopus (2015). Scopus. Recuperado de http://www.scopus.com/results/results. url? numberOfFields $=0 \& s r c=s \&$ clickedLink $=$ \&edit $=$ \&editSaveSearch $=\&$ origin = searchbasic \&authorTab $=\&$ affiliationTa $b=\&$ advancedTa $b=\&$ scint $=1 \&$ menu=search\&tablin=\&searchterm $1=$ Tourism\&field 1=TITLE_ABS_KEY\&dateType=Publication_Date_Type\&yearFrom=Before+1960\&yearTo=Present\&loadDate $=7 \&$ documenttype $=$ All \&subjects $=$ LFSC \&subjects $=$ HLSC\&subjects $=$ PHS $C \&$ subjects $=S O S C \& s r c=s \& s t 1=$ Tourism \& st $2=\& s o t=b \& s d t=b \& s I=\& s=T I T-$ LE-ABS-KEY\%28Tourism\%29\& sid=BF59D5B654C6A0C095AEA 95EB734CE64. FZg2ODcJC9ArCe8WOZPvA\%3A10\&searchld=BF59D5B654C6A0C095AEA95EB734CE64.FZg2ODcJC9ArCe8WOZPvA\%3A 10\&txGid=BF59D5B654C6A0C095AEA95EB734CE64.FZg2ODcJC9ArCe8WOZPvA\%3A1\&sort=plf-f\&originationType $=b \& r r=\&$ null $=$.

Serres, M. (1991). El Paso del Noroeste. Hermes V. Madrid, España: Debate.

Sharpley, R. (2011). The Study of Tourism: Past Trends and Future Directions. Contemporary Geographies of Leisure, Tourism and Mobility. Abingdon, Estados Unidos: Routledge.

Thirkettle, A. y Korstanje, M. (2013). Creating a new epistemology for tourism and hospitality disciplines. Int. J. Qualitative Research in Services, 1(1), 13-34.

Tribe, J. (2000). Indisciplined and unsubstantiated. Annals of Tourism Research, 27(3), 809813.

Tribe, J. (2006). The truth about tourism. Recuperado de http:/ /epubs.surrey.ac.uk/1127/1/ fulltext.pdf.

Tribe, J. (2010). Tribes, territories and networks in the tourism academy. Annals of Tourism Research, 37(1), 7-33.

Turner, L. y Ash, J. (1991). La Horda Dorada. Madrid, España: Ediciones Endymion.

Universidad Nacional Autónoma de México (2007). Elaboración de un Diagnóstico del Estado de la Investigación Turística en el País y Generación de un Programa de Investigación Integral para el Sector Turismo. Ciudad de México, México: Secretaría de Turismo.

Urry, J. (2004). La mirada del turista. Lima, Perú: Universidad de San Martín de Porres.

Vanhove, N. (2011). The Economics of Tourism Destinations. Abingdon, Estados Unidos: Routledge.

Vogel, H. L. (2001). Travel Industry Economics. Cambridge, Reino Unido: Cambridge University Press.

World Tourism Organization (2015). Annual Report 2014. Madrid, España: Autor.

Xiao, H. y Smith, S. (2006). The making of tourism research. Annals of Tourism Research, 33(2), 490-507.

Xiao, H. y Smith, S. (2007). The use of tourism knowledge. Research propositions. Annals of Tourism Research, 34(2), 310-331. 